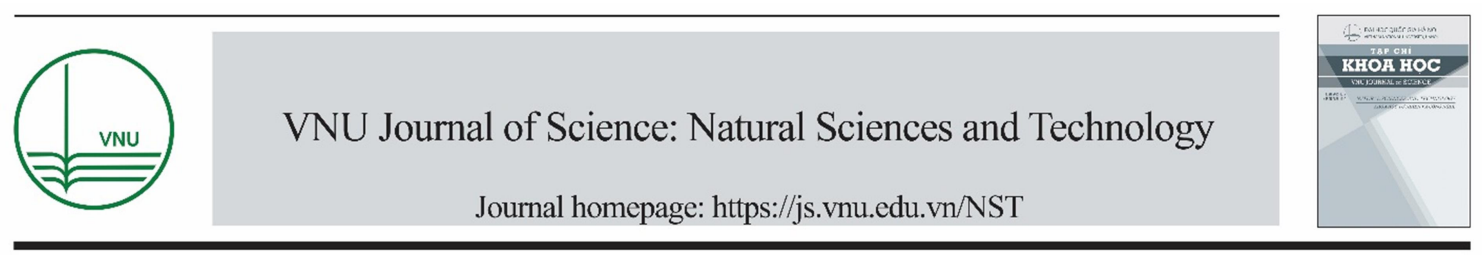

\author{
Original Article
}

\title{
Sequencing Coding Region and in Silico Structural Analysis of Protein Hexon of HAdV-3 Causing Conjunctivitis in Vietnam
}

\author{
Nguyen Van Sang ${ }^{*}$, Nguyen Thi Thu Huyen, Le Tuan Anh \\ VNU University of Science, 334 Nguyen Trai, Hanoi, Vietnam \\ Received 22 April 2020 \\ Revised 25 November 2020; Accepted 27 November 2020
}

\begin{abstract}
Human adenovirus (HAdVs) are responsible for about $65-90 \%$ of viral conjunctivitis. Understandings of HAdVs bring promises in prevention, treatments, and biological preparations. However, thorough researches on structural proteins of HAdVs are still limited in Vietnam. In this study, we have sequenced the entire coding gene of protein hexon from HAdV-3 causing conjunctivitis in Vietnam and compared with the reference sequence in NCBI database. We detected 42 DNA variants, of which, 11 resulted in amino acid substitutions. Simulation of HAdV3 hexon structure showed that all 11 substitutions located in crucial positions. This result revealed high risks from hexon gene variants of HAdV-3 enhancing its life span, virulence, and ability to avoid the host immune system.
\end{abstract}

Keywords: Conjunctivitis, HAdV-3, Hexon structure

\section{Introduction}

To date, HAdVs includes roughly 100 different types belonging to family Adenoviridae, genera Mastadenovirus, which are divided into 7 species represented by letters from A to $G[1,2]$. The capsid of HAdVs consists of 20 facets made up of 3 main proteins hexon, penton, and fiber. Therein, hexon constitutes the major part of virus coat protein

* Corresponding author.

Email address: nvsangvnu@gmail.com

https://doi.org/10.25073/2588-1140/vnunst.5064 with 12 homo-trimer hexon proteins per facet [3]. The faceted exterior of hexon introduces 7 hypervariable regions (HVRs) in loop-1 and loop-2 structures that consider characteristics for HAdVs genotyping [4,5]. Therefore, researching the structure of hexon protein is important to explore the immune adaptation and develop novel biological applications. Understandings about HAdVs have been worldwide employed in vaccine producing (development) [6] and cancer therapies [7,8]. In Vietnam, there was one research that used HVR-7 to classify different HAdV types associated with conjunctivitis; and HAdV-3 
was one of the types circulating in Hanoi, Vietnam [9]. In this study, we focused on sequencing the coding region and analyzing the 3D structure of hexon protein of HAdV-3 isolated in Vietnam; and then, evaluating the consequences of amino acid substitutions to the life span and virulence of the virus.

\section{Materials and methods}

\subsection{Collecting samples}

The eye-swabs of voluntary conjunctivitis patients were collected at Vietnam National Institute of Ophthalmology. The samples were kept in $1.5 \mathrm{ml}$ tubes and stored at $-20^{\circ} \mathrm{C}$ until used for viral DNA extraction.

\subsection{Viral DNA extraction and detecting the presence of $H A d V-3$}

The genome of HAdVs was purified by using Viral Gene-Spin Virus RNA/DNA

Table 1. Sequence of primers used to amplify hexon gene of HAdV-3 in Vietnam

\begin{tabular}{llc}
\multicolumn{1}{c}{ Primers } & \multicolumn{1}{c}{ Sequence (5'-3') } & Amplicon size (bp) \\
\hline HF1 & CTTAATGACTGTTGACGCTG & 4274 \\
\hline HR1 & GGATCAAAAAGGTAGCAGGT & \multirow{2}{*}{3131} \\
\hline HF2 & CTCTGGTATTAACGGCGTAG & \\
\hline HF3 & GTATGGATAATTGGCTGGGT & 1878 \\
\hline HR3 & GCTTAACTTGCTTGTCTGTG & \\
\hline
\end{tabular}

\subsection{PCR components and thermal cycles}

All of the amplifying reactions were carried out with enzyme Phusion Hotstart II DNA Polymerase (Thermo Scientific). Each $50 \mu \mathrm{l}$ PCR reaction mix contained $10 \mu \mathrm{l}$ of GC buffer (5X), $1.5 \mu 1$ of each forward and reverse primer (10pmol $\left.\mu \mathrm{l}^{-1}\right), 1 \mu \mathrm{d} \mathrm{dNTPs} \operatorname{mix}(10 \mu \mathrm{M}$ each), $1.5 \mu 1$ DMSO (100\%), 0.5 ul enzyme $(2 \mathrm{U} / \mu \mathrm{l})$ and $1 \mu \mathrm{l}$ of sample template. Amplifications included $30 \mathrm{~s}$ initial denaturation at $98^{\circ} \mathrm{C}$, followed by 40 cycles at $98^{\circ} \mathrm{C}(20 \mathrm{~s}), 60^{\circ} \mathrm{C}(10$ s), and $72^{\circ} \mathrm{C}(1-3 \mathrm{~min})$, with a final extension at $72^{\circ} \mathrm{C}$ (5 min). Annealing temperature was
Isolation Kit manufactured by iNtRon company (Korea). Viral DNA elution was stored at $20^{\circ} \mathrm{C}$, ready for PCR. Then, we amplified and sequenced the HVR-7 region to detect the presence of HAdV-3 in the samples. The methods were described in more detail by $\mathrm{Ha}$, et al. [9].

\subsection{Designing primers for amplifying hexon coding region}

We collected and aligned the HAdV-3 genomes available in NCBI database to find the conserved regions flanking hexon gene. The first primer pair HF1-HR1 was designed based on the conserved regions. Then, like the primer walking technique, the second primer pair HF2HR2 was designed on 3'-terminator region of the first sequences. Similarly, with primer pair HF3-HR3, we could obtain the full coding sequence of hexon gene of HAdV-3 (Table 1) 
by BigDye ${ }^{\circledR}$ Terminator v3.1 cycle sequencing kit.

\subsection{Analysis of DNA sequence}

Sequencing results were read and edited on BioEdit program [10]. The coding sequence of hexon gene of HAdV-3 in Vietnam was aligned with reference sequence NC_011203.1 by ClustalW program [11].

\subsection{Structural analysis of hexon protein}

The DNA sequence was translated to amino acid sequence (1 letter code) in Snapgene software then align with 9 other hexon protein sequences from 7 different HAdV types. The putative 3D structure of hexon of HAdV-3 in Vietnam was constructed by Swiss-Model program [12], based on its amino acid sequence and data in Protein Data Bank (PDB). The obtained structure was performed on pyMOL software [13].

\section{Results and discussion}

\subsection{PCR amplification of designed primer pairs}

The length of hexon gene was $2835 \mathrm{bp}$. However, the amplicon obtained from PCR with HF1-HR1 primers was $4274 \mathrm{bp}$ long. The desired bands for PCR of HF2-HR2 and HF3HR3 primer pairs were 3131 bp and 1878 bp. The sequences of primers are described in Table 1. The electrophoresis results in Figure 1 showed that the desired bands were as bright as the marker and no undesired bands were observed. Thus, the PCR products were used for sequencing reactions.
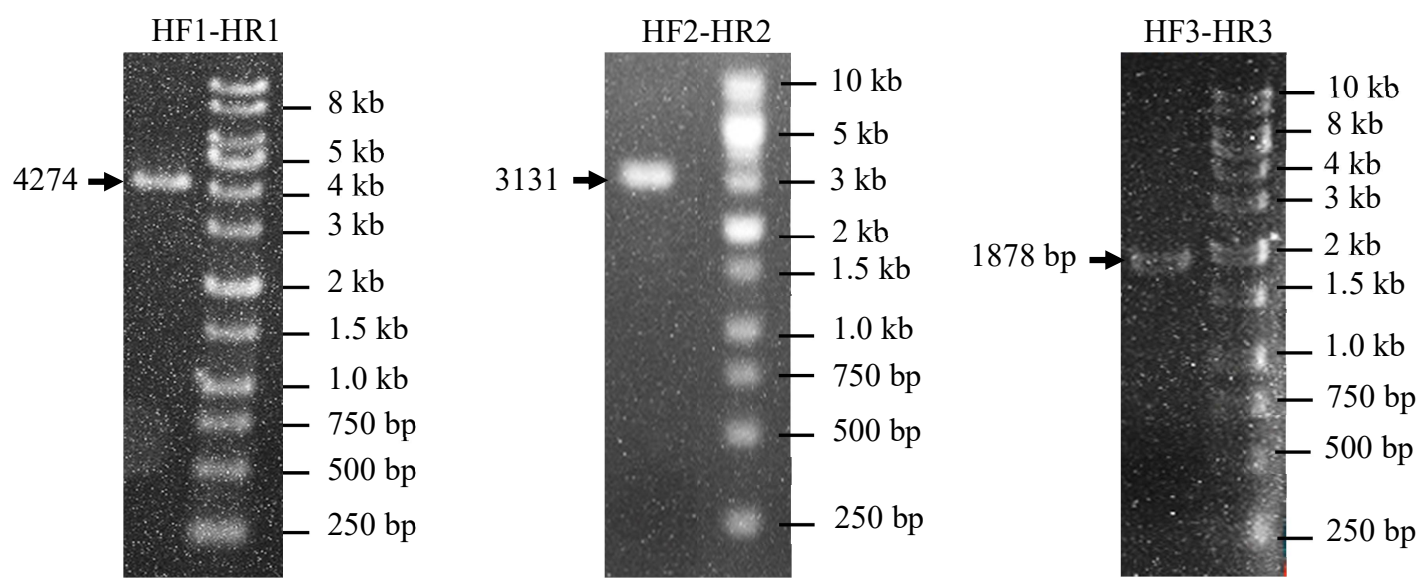

Figure 1. The PCR-amplified products of hexon gene of HAdV-3 in Vietnam with respective primer pairs

\subsection{Analysis of DNA sequence}

We obtained the full coding sequence of hexon gene of conjunctivitis-induced HAdV-3 in Vietnam. The residual sequences were cut out in BioEdit program. All of the sequencing signals were well resolved with high and clear peaks (Figure 2). 


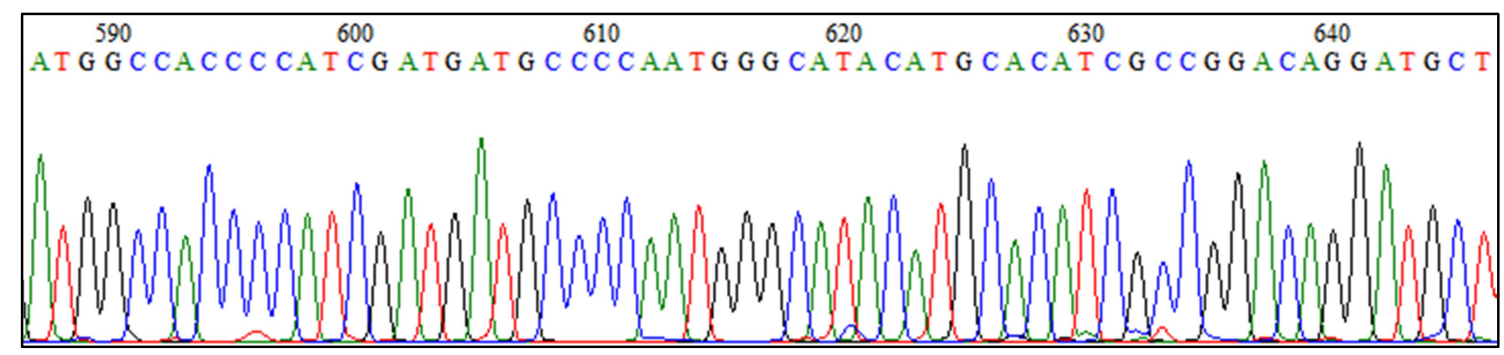

Figure 2. Signals of the first 60 nucleotides of hexon gene of HAdV-3 in Vietnam

We aligned our sequence with the reference sequence of HAdV-3 in the Genbank database (accession number: NC_011203) and detected 42 DNA variants scattering along hexon gene (Table 2), in which, 11 DNA variants led to amino acid substitutions. Nine out of 11

Table 2. DNA variants led to amino acid changes in hexon sequence of HAdV-3 in Vietnam

\begin{tabular}{|c|c|c|c|c|c|c|c|c|c|}
\hline \multirow{2}{*}{$\mathbf{N}^{\mathbf{0}}$} & \multirow{2}{*}{ Position } & \multicolumn{2}{|c|}{ Nucleotide } & \multirow{2}{*}{$\begin{array}{l}\text { Amino Acid } \\
\text { change }\end{array}$} & \multirow{2}{*}{$\mathbf{N}^{\mathbf{0}}$} & \multirow{2}{*}{ Position } & \multicolumn{2}{|c|}{ Nucleotide } & \multirow{2}{*}{$\begin{array}{l}\text { Amino acid } \\
\text { changes }\end{array}$} \\
\hline & & $\mathrm{Seq}^{1}$ & $\mathrm{Seq}^{2}$ & & & & $\mathrm{Seq}^{1}$ & $\mathrm{Seq}^{2}$ & \\
\hline 1 & 18489 & $\mathrm{C}$ & $\mathrm{G}$ & - & 22 & 20085 & G & A & - \\
\hline 2 & 18542 & G & $\mathrm{C}$ & G42A & 23 & 20103 & G & A & - \\
\hline 3 & 18594 & $\mathrm{C}$ & $\mathrm{T}$ & - & 24 & 20149 & $\mathrm{C}$ & A & - \\
\hline 4 & 18834 & A & $\mathrm{G}$ & - & 25 & 20202 & G & A & - \\
\hline 5 & 18838 & $\mathrm{G}$ & $\mathrm{C}$ & G141R & 26 & 20439 & A & G & - \\
\hline 6 & 18840 & G & A & - & 27 & 20529 & $\mathrm{~T}$ & $\mathrm{C}$ & - \\
\hline 7 & 19313 & A & $\mathrm{G}$ & E299G & 28 & 20610 & $\mathrm{~T}$ & $\mathrm{C}$ & - \\
\hline 8 & 19321 & A & G & N302D & 29 & 20694 & G & A & - \\
\hline 9 & 19380 & $\mathrm{C}$ & A & - & 30 & 20712 & $\mathrm{~T}$ & $\mathrm{C}$ & - \\
\hline 10 & 19395 & $\mathrm{C}$ & $\mathrm{T}$ & - & 31 & 20856 & $\mathrm{C}$ & $\mathrm{T}$ & - \\
\hline 11 & 19648 & A & $\mathrm{G}$ & N411D & 32 & 20883 & G & A & - \\
\hline 12 & 19670 & $\mathrm{C}$ & $\mathrm{G}$ & \multirow{2}{*}{ T418R } & 33 & 20892 & A & G & - \\
\hline 13 & 19671 & A & $\mathrm{G}$ & & 34 & 20964 & G & A & - \\
\hline 14 & 19702 & A & $\mathrm{G}$ & T429A & 35 & 20970 & $\mathrm{C}$ & $\mathrm{T}$ & - \\
\hline 15 & 19733 & $\mathrm{C}$ & A & A439D & 36 & 20976 & $\mathrm{G}$ & A & - \\
\hline 16 & 19735 & $\mathrm{C}$ & A & $\mathrm{P} 440 \mathrm{~T}$ & 37 & 21012 & $\mathrm{~T}$ & A & - \\
\hline 17 & 19750 & A & G & $\mathrm{T} 445 \mathrm{~A}$ & 38 & 21015 & $\mathrm{C}$ & G & - \\
\hline 18 & 19758 & $\mathrm{C}$ & A & - & 39 & 21042 & $\mathrm{G}$ & A & - \\
\hline
\end{tabular}

substitutions were observed to locate in hypervariable regions of hexon protein (HVR-1 to 7) $[14,15]$. Meanwhile, no variants that cause insertion, deletion, or frameshifts were observed. 


\begin{tabular}{llllllllll}
$\mathbf{1 9}$ & 19818 & $\mathrm{~T}$ & $\mathrm{C}$ & - & $\mathbf{4 0}$ & 21051 & $\mathrm{C}$ & $\mathrm{G}$ & - \\
\hline $\mathbf{2 0}$ & 19929 & $\mathrm{C}$ & $\mathrm{T}$ & - & $\mathbf{4 1}$ & 21067 & $\mathrm{~A}$ & $\mathrm{C}$ & M884L \\
\hline $\mathbf{2 1}$ & 19953 & T & C & - & $\mathbf{4 2}$ & 21084 & $\mathrm{~A}$ & $\mathrm{G}$ & - \\
\hline
\end{tabular}

Reference sequence of HAdV-3 (NC 011203)

${ }^{2}$ The sequence of HAdV-3 sample in Vietnam

"-” represented no changes of amino acid in this site

\subsection{Analysis of protein structure}

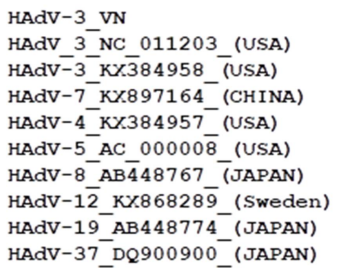

HAdV-3 VN

HAdV 3 NC 011203 (USA) HAdV-3_KX3384958_(USA) HAdV-7_KX897164_(CHINA) HAdV $-4{ }_{-}^{-}$KX384957_(USA) HAdV -5 - AC_ $00000 \overline{8}_{-}$(USA) HAdV-8_AB 4 48767 (JAPAN) HAdV-12_KX868289_ (Sweden) HAdV-19_AB 448774_(JAPAN) HAdV-37_DQ900900_(JAPAN)

HAdV-3_VN

HAdV_3_NC_011203_(USA) HAdV $-3 \times \times 384958$ (USA) HAdV $-7^{-}$KX897164 (CHINA) HAdV-4_KX384957_(USA) HAdV -5 - AC_00000 $\overline{8}$ (USA) HAdV -8 AB4 48767 (JAPAN) HAdV $-1 \overline{2}$ KX86828 $\overline{9}$ (Sweden) HAdV $-19^{-}$AB 4 48774- (JAPAN) HAdV-37_DQ900900_(JAPAN)

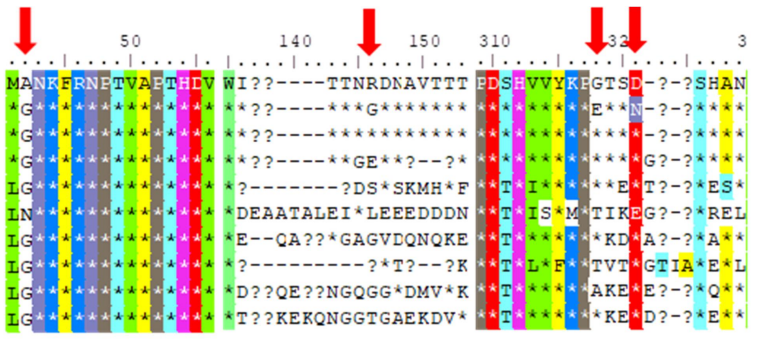

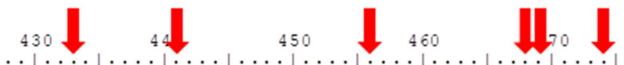
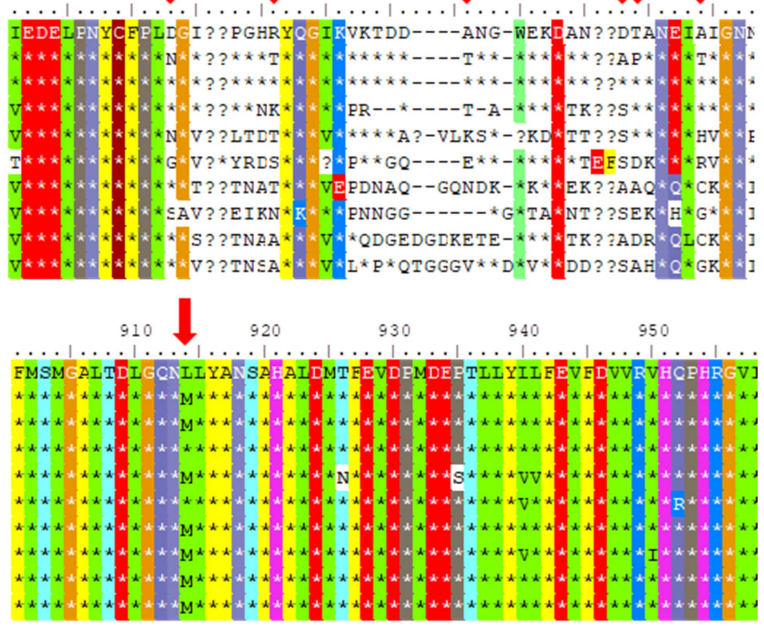

Figure 3. Comparison of hexon protein sequences of HAdVs from different HAdV species

Ten sequences of hexon protein were aligned by ClustalW method using BioEdit software. The result shows the 11 positions of amino acid changes in HAdV-3 VN (red arrow) which compared with type HAdV-3 (NC_O1 1203). Most of them belong to HVR regions (no color).

The prior researches showed evidence that the host immune system recognizes and terminates HAdVs through interactions with hexon [18, 19]; we assume, those 9 substitutions on the HVR regions, have high chances of helping the virus avoid the immune activities in Vietnamese patients. Figure 3 showed that most of those substitutions belong to HVR regions. Thus, we predict the 3D structure model of our amino acid sequence to study the meaning of changes for those substitutions. 


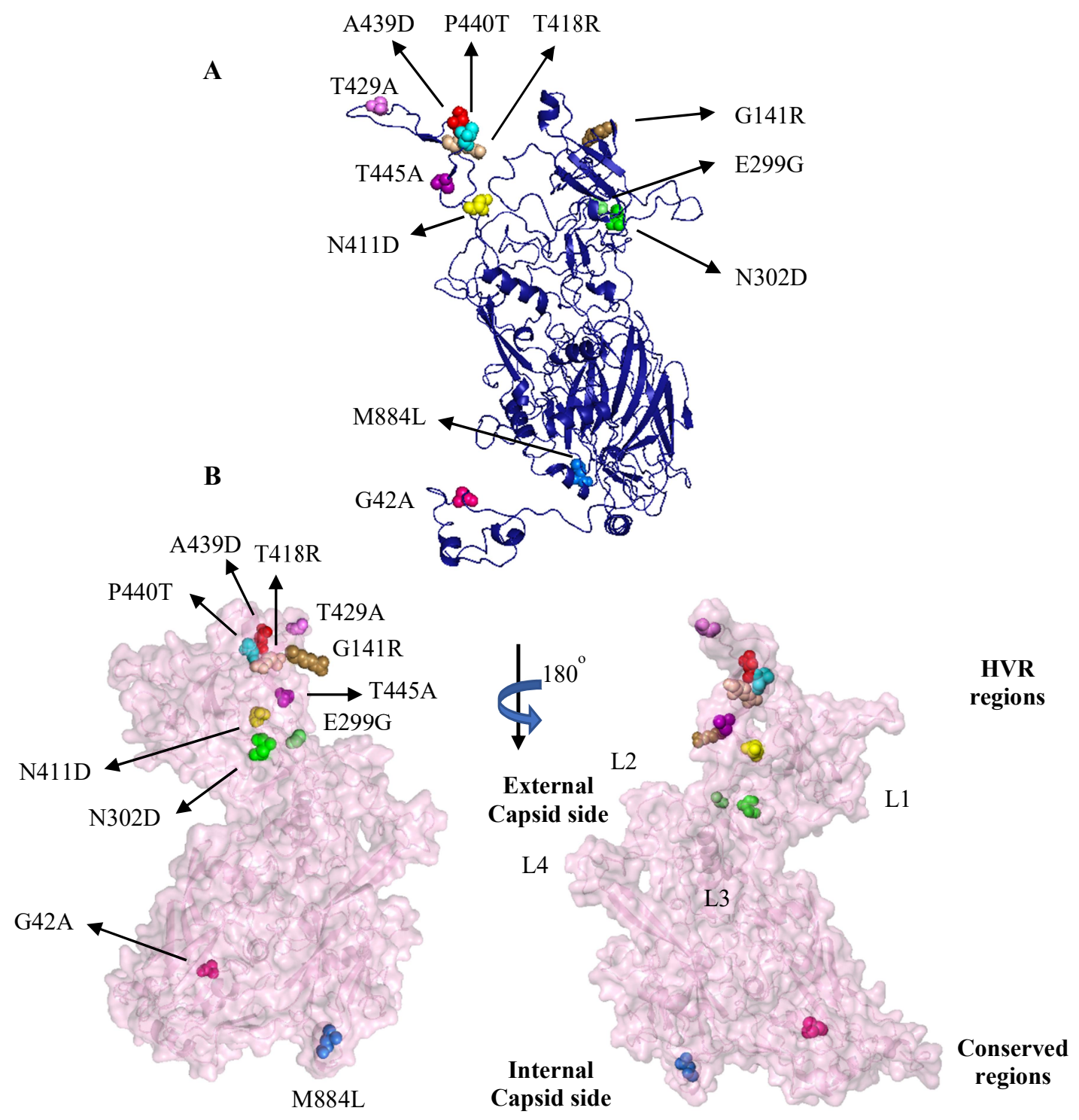

Figure 4. Structure of mono-hexon protein of HAdV-3 VN

Side views of predicted $3 D$ model of HAdV-3 VN mono-hexon protein based on the cryo-EM structure of HAdV-26 (PDB; 5TX1). (A) Cartoon structure of HAdV-3 VN with amino acid substitutions depicted on monohexon structure (sideview). (B) The protein surface was represented by 0.65 cartoon transparency. All structure models exposed 11 amino acid changes with highlight by sphere representation as G42A color hot pink, G141R color sand, E299G color lime, N302D color green, N411D color yellow, T418R color wheat, T429A color violet, A439D color red, P440T color cyan, T445A color purple, M884L color marine. L1-14 represent loop 1- loop 4 of hexon protein.

The Swiss-Model program founds 22 structural templates that can be used to build a
3D model of hexon of HAdV-3 in Vietnam. In which, 2OBE [16] has the highest identity to 
our sequence $(87.11 \%)$. However, 2OBE was hexon of Chimpanzee Adenovirus and the protein was described only in the monomer state. Therefore, we chose 5TX1 [17] as the template to build the putative model for hexon of HAdV-3 in Vietnam (identity $84.28 \%$, HAdV-26). All of 11 amino acid substitutions were depicted on putative hexon structure in PyMOL software. Whereby, G42A and M884L located on the inner side (conserved region) of the capsid while the other 9 substitutions were on the outer side (HVR regions) (Figure 4).

Substitution M884L was found in the interacting sites among hexon homo-trimers (Figure 5). Leucine (L) is highly hydrophobic while Methionine (M) is significantly less hydrophobic, which might make bonds among hexon homo-trimers stronger, thus the capsid

$\mathbf{A}$

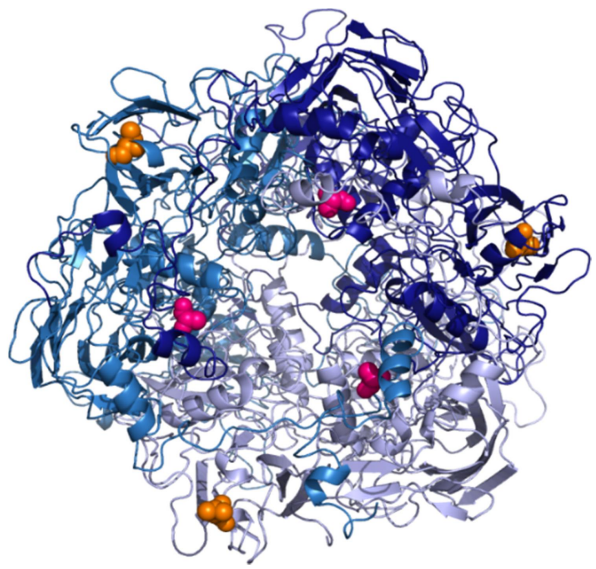

would be more stable and solid. The last substitution - G42A sited in the central region of each homo-trimer hexon where protein VI clings to (protein VI plays a vital role in cleaving precursor proteins) $[20,21]$. Since a neutral amino acid-like Glycine (G) was replaced by Alanine (A) - a hydrophobic amino acid, we suppose the G42A substitution affected the binding ability of protein VI to hexon, stabilized interactions and enhanced virulence of the virus.

Besides, our results showed that the regions forming $\alpha$ helices and $\beta$ sheets of hexon of HAdV-3 in Vietnam were highly conserved. This agrees with other researches showing that hexon structures of different types: HAdV-26, HAdV-5, and HAdV-2 were almost identical, except HVR regions [17, 22].

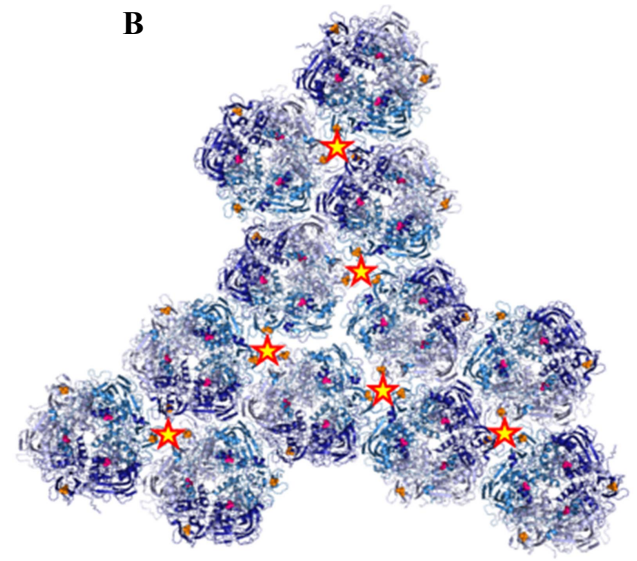

Figure 5. Homo-trimer hexon (view from inside)

A. Substitutions G42A (pink) and M884L (orange). B. 12 homo-trimer hexon proteins make up a facet, M884L substitutions join at "star" signs.

\section{Conclusions}

We successfully sequenced the coding region of hexon of HAdV-3 causing conjunctivitis in Vietnam. We detected 42 DNA variants throughout the gene, in which, 31 were silent mutations and 11 led to amino acid substitutions. Nine substitutions were determined to locate on HVRs of the capsid, having potentials in helping the virus escaping the immune system. Meanwhile, two substitutions (G42A and M884L) are anticipated to enhance the vitality and virulence of HAdV-3 in Vietnam. Lastly, the putative structure of hexon of HAdV-3 in Vietnam was determined to be conserved with other HAdV types in the world. 


\section{Acknowledgments}

This research is funded by the Vietnam National University, Hanoi (VNU) under project number QG.17.19

\section{References}

[1] D. Seto, J. Chodosh, J. R. Brister, M. S. Jones, C. Members of the Adenovirus Research, Using the whole-genome sequence to characterize and name human adenoviruses, Journal of virology. 85 (2011) 5701-5702. https://doi.org/10.1128/JVI.00 354-11.

[2] D. Seto, J. R. Brister, J. Chodosh, D. T. Curiel, A. Heim, M. S. Jones, A. Kajon, T. Lion, Q. Zhang, Human-Adenovirus-Working-Group.

http://hadvwg.gmu.edu/. July, 2019.

[3] W. Chiu, R. M. Burnett, R. L. Garcea, Structural biology of viruses, Oxford University Press, New York, 1997. https://books.google.com.vn/books? id=KUgQAQAAMAAJ.

[4] L. K. Crawford-Miksza, D. P. Schnurr, Adenovirus Serotype Evolution Is Driven by Illegitimate Recombination in the Hypervariable Regions of the Hexon Protein, Virology. 224 (1996) 357-367. https://doi.org/10.1006/viro.1996 .0543 .

[5] J. G. D. Gall, R. G. Crystal, E. Falck-Pedersen, Construction and Characterization of HexonChimeric Adenoviruses: Specification of Adenovirus Serotype, Journal of Virology. 72 (1998) 10260. https://doi.org/10.1128/JVI.72.12.1 0260-10264.1998.

[6] C. Deal, A. Pekosz, G. Ketner, Prospects for oral replicating adenovirus-vectored vaccines, Vaccine. 31 (2013) 3236-3243. https://doi.org/10 .1016/j.Vaccine.2013.05.016.

[7] S. Pesonen, L. Kangasniemi, A. Hemminki, Oncolytic Adenoviruses for the Treatment of Human Cancer: Focus on Translational and Clinical Data, Molecular Pharmaceutics. 8 (2011) 12-28. https://doi.org/10.1021/mp100219n.

[8] M. Schmid, P. Ernst, A. Honegger, M. Suomalainen, M. Zimmermann, L. Braun, S. Stauffer, C. Thom, B. Dreier, M. Eibauer, A. Kipar, V. Vogel, U. F. Greber, O. Medalia, A. Plückthun, Adenoviral vector with shield and adapter increases tumor specificity and escapes liver and immune control, Nature Communications. 9 (2018) 450. https://doi.org /10.1038/s41467-017-02707-6.
[9] N. V. Ha, N. T. T. Huyen, D. T. T. Huyen, N. Q. Hung, T. T. Anh, H. A. Tuan, N. V. Sang, Method Development for Detection and Classification of Conjunctivitis-Causing Adenoviruses in Human, VNU Journal of Science: Natural Sciences and Technology. 32 (2016)

[10] T. A. Hall, BioEdit : a user-friendly biological sequence alignment editor and analysis program for Windows 95/98/NT, Nucleic Acids Symp. Ser. 41 (1999) 95-98.

[11] J. D. Thompson, D. G. Higgins, T. J. Gibson, CLUSTAL W: improving the sensitivity of progressive multiple sequence alignment through sequence weighting, position-specific gap penalties and weight matrix choice, Nucleic Acids Research. 22 (1994) 4673-4680. https://doi.org/1 $0.1093 / \mathrm{nar} / 22.22 .4673$.

[12] A. Waterhouse, M. Bertoni, S. Bienert, G. Studer, G. Tauriello, R. Gumienny, F. T. Heer, T. A. P. de Beer, C. Rempfer, L. Bordoli, R. Lepore, T. Schwede, SWISS-MODEL: homology modelling of protein structures and complexes, Nucleic Acids Research. 46 (2018) W296-W303. https://doi.org/10.1093/nar/gky427.

[13] W. L. DeLano, The PyMOL Molecular Graphics System, Educationnal Version Schrödinger, LLC, (2002)

[14] L. Crawford-Miksza, D. P. Schnurr, Analysis of 15 adenovirus hexon proteins reveals the location and structure of seven hypervariable regions containing serotype-specific residues, Journal of Virology. 70 (1996) 1836-1844. https://doi.org /10.1128/JVI.70.3.1836-1844.1996.

[15] J. Rux, R. Burnett, Type-Specific Epitope Locations Revealed by X-Ray Crystallographic Study of Adenovirus Type 5 Hexon, Molecular therapy : the journal of the American Society of Gene Therapy. 1 (2000) 18-30. https://doi.org/10.1006/mthe.1999.0001.

[16] S. L. Pichla-Gollon, M. Drinker, X. Zhou, F. Xue, J. J. Rux, G.-P. Gao, J. M. Wilson, H. C. J. Ertl, R. M. Burnett, J. M. Bergelson, Structure-based identification of a major neutralizing site in an adenovirus hexon, Journal of virology. 81 (2007) 1680-1689. https://doi.org/10.1128/JVI.02023-06.

[17] X. Yu, D. Veesler, M. G. Campbell, M. E. Barry, F. J. Asturias, M. A. Barry, V. S. Reddy, CryoEM structure of human adenovirus D26 reveals the conservation of structural organization among human adenoviruses, Science Advances. 3 (2017) https://doi.org/10.1126/sciadv.1602670.

[18] S. Roy, P. S. Shirley, A. McClelland, M. Kaleko, Circumvention of immunity to the adenovirus 
major coat protein hexon, Journal of Virology. 72 (1998) 6875-6879. https://doi.org/10.1128/JVI.7 2.8.6875-6879.1998.

[19] C. Wohlfart, Neutralization of adenoviruses: kinetics, stoichiometry, and mechanisms, Journal of Virology. 62 (1988) 2321-2328.

[20] H. Liu, L. Jin, S. B. S. Koh, I. Atanasov, S. Schein, L. Wu, Z. H. Zhou, Atomic structure of human adenovirus by cryo-EM reveals interactions among protein networks, Science (New York, N.Y.). 329 (2010) 1038-1043. https://doi.org/10.1126/science.1187433.
[21] C. M. Wiethoff, H. Wodrich, L. Gerace, G. R. Nemerow, Adenovirus protein VI mediates membrane disruption following capsid disassembly, Journal of Virology. 79 (2005) 1992-2000. https://doi.org/10.1128/JVI.79.4.19 92-2000.2005.

[22] J. J. Rux, P. R. Kuser, R. M. Burnett, Structural and Phylogenetic Analysis of Adenovirus Hexons by Use of High-Resolution X-Ray Crystallographic, Molecular Modeling, and Sequence-Based Methods, Journal of Virology. 77 (2003) 9553. https://doi.org/10.1128/JVI .77.17.9553-9566.2003. 